\title{
8 Using the history of mathematics as a source of technology-rich problems
}

\author{
Jerry Johnson \\ Western Washington University \\ Bellingham, Washington, USA
}

\begin{abstract}
Computing technologies can help motivate students to explore problems and topics from the history of mathematics. Because the activities are project based, the role of the teacher becomes that of a resource. Modified assessment techniques must be implemented. On the part of both the teacher and the students, the end results are increased mathematical understanding as well as a better appreciation of both how mathematical ideas developed historically and the individuals behind these developments.
\end{abstract}

\section{Keywords}

Pedagogy, problem solving, interdisciplinary, computing, ethnomathematics.

\section{Introduction}

Unfortunately, most mathematics teachers and secondary school students fall far short in their understanding of how mathematical ideas developed historically as well as the individuals behind these developments. It is important that they gain some sense of the impact of the concrete physical world, the interdisciplinary contexts, the role of powerful intuitions, and the growth of mathematical abstraction. Access to computing technologies provides both a means and a motivation for student exploration of topics in the history of mathematics. Furthermore, these technological explorations can easily be integrated into an already crowded curriculum.

In addition to graphing calculators, the computer technology necessary for these explorations include spreadsheets, dynamic geometry software (e.g. Cabri, The Geometer's Sketchpad), symbolic algebra systems (e.g. Mathematica, Maple, Derive), 2D/3D graphing packages, and simulation software (e.g. Stella). Also, proficiency in one or more programming languages (e.g. Pascal, $\mathrm{C}++, \mathrm{Logo}$ ) will allow the design of more specific investigations.

Students can communicate the results of their investigations in the form of CD-ROM multi-media presentations, World Wide Web pages on the Internet, and data bases. These communications should include a historical context, an 
overview of the historical personalities involved, a summary of the original problem or idea, and a full presentation of the student's investigations.

The student's first step is to find an interesting problem or idea that is accessible, has a rich historical context, and has the potential for creative investigation. Any of the general reference texts on the history of mathematics can serve as the source of these problems or ideas. Other resources are anthologies of original writings of mathematicians or reference texts that focus on the history of a specific mathematical topics.

\section{Process}

The student will spend a considerable amount of time trying to understand the original problem or idea, due to changes in notation and context. Computing technologies can help gain this understanding. Often, the mathematics teacher will serve as an interactive resource, and possibly be pushed mathematically as well. At this stage of the investigation, the assumption that old mathematics is trivial mathematics is easily proven false.

In the further investigation of the problem or idea, the student can show considerable creativity. A minimal expectation is the use of computing technologies to demonstrate understanding of the mathematics involved in its original historical context. The motivated student may move beyond this minimal expectation to the actual doing of new mathematics by investigations of extensions of the problem or ideas involved. Hindsight and access to the power of modern mathematical techniques empower the student in these extensions.

\section{Examples}

In the compilation of examples of potential explorations, an attempt was made to include problems or ideas representative of a broad range of mathematical content and the full history of mathematics. The following list of 25 examples is certainly not exhaustive.

- Comparison of Babylonian, Greek, and Chinese solutions of quadratics, including root approximations and the rule of double false position;

- Pythagorean number theory, including figurate numbers, amicable numbers, and Pythagorean triples;

- Khayyam's solution of the cubics;

- Three famous construction problems: duplication of the cube, trisection of an angle, and quadrature of a circle;

- Techniques for the computation of values of pi;

- Geometrical excursions such as the Lunes of Hippocrates, Archimedes shoemaker's knife, Pappus' anharmonic ratio;

- Euclidean constructions, such as the process of divisions and the use of collapsible compasses;

- Apollonius' conic sections or three circle problem;

- Diophantine problems connecting algebra and number theory, 
- Map projections of a sphere onto a plane;

- Fractal geometry, such as the Koch curve, the Mandelbrot set, and connections to Pascal's triangle;

- Pascal's and Galileo's investigations of a cycloid and its analogues, including the Bernoulli's work on the brachistochrone problem;

- Descartes' techniques for constructing tangents to a curve;

- Fermat's conjectures in number theory and the method of infinite descent;

- Probability problems posed by Huygens, Fermat, and Pascal;

- Newton's method and its refinements;

- Euler's investigations in number theory,

- Lagrange's Theorem in group theory,

- Buffon's needle problem and Laplace's extension;

- Gauss' theory of congruence and modulo arithmetic;

- Fourier series and applications;

- Models for non-euclidean geometries (e.g. Klein or Poincare);

- Contributions to projective geometry by Desargues, Poncelet, Steiner, and Mőbius;

- Monge's contributions to differential geometry,

- The search for large prime numbers.

\section{Implementation and Assessment.}

These projects can easily be dove-tailed with on-going classroom instruction, with ample time and access to computer resources outside of class being required on the part of both the students and their teacher. Because the projects require an extended time-period, a time-schedule should be established at the very beginning and closely monitored by the teacher. Furthermore, a mechanism needs to be implemented for monitoring progress, and possibly linked to a system of assessment. Key partitions are the selection of the problem, development of understanding of the problem and its context, further investigation of extensions of the problem, and communication of student results. In order for each student to achieve success, the teacher must carefully monitor the student's original problem selection, using criteria such as the student's ability in mathematics, use of computing technologies, and ability to work independently. It is possible for students to work in small groups on these projects, though student roles and responsibilities need to be clearly identified.

Assessment of the project must reflect all of the project's aspects. That is, the assessment must include the selection of the original problem (e.g. appropriateness, level of mathematical sophistication), the development of a historical context, the demonstration of an understanding of the original problem, the use of computing technologies, the investigation of extensions of the problem, and the communication of final results. The biggest difficulty is the inclusion of the assessment of this project into the overall assessment of the student for the ongoing mathematics course. 